\title{
Avaliação neuropsicológica na encefalopatia de Hashimoto: Um relato de caso
}

\author{
Evaluación neuropsicológica en la encefalopatía de Hashimoto: un estudio de caso \\ Évaluation neuropsychologique de l'encéphalite d'Hashimoto: une étude de cas \\ Neuropsychological assessment in Hashimoto's encephalitis: A case report
}

\section{Tatiana Q. Irigaray ${ }^{1}$, Michele Beckert ${ }^{2}$, Angélica D. Pizzol², Candice S. Holderbaum² ${ }^{2}$, Denise R. Fontoura ${ }^{2}$, Thirzá B. Frison², Carlo M. Mattiello², Taís P. Fachinelli², Márcia L. F. Chaves², Marino M. Bianchin ${ }^{2}, \&$ Jerusa F. Salles ${ }^{2}$}

\author{
${ }^{1}$ Pontifícia Universidade Católica do Rio Grande do Sul, Brasil. ${ }^{2}$ Universidade Federal do Rio Grande do Sul, Brasil.
}

Resumo

\begin{abstract}
A Encefalopatia de Hashimoto (EH) é uma condição neurológica rara, que está associada à presença de altas concentrações de anticorpos anti-tireoideanos e à tireoidite de Hashimoto. Nesse artigo, relata-se o caso de uma paciente de 60 anos com diagnóstico de EH, enfatizando o seu desempenho em testes neuropsicológicos antes e após tratamento medicamentoso. Ela era previamente hígida, mas seis meses antes do diagnóstico, começou a apresentar depressão, crises epilépticas, confusão mental, apraxias, mioclonias e alucinações visuais. Antes do tratamento, foi submetida a uma avaliação neuropsicológica que evidenciou déficits cognitivos globais. Seis meses após tratamento, ela foi reavaliada, mostrando uma recuperação cognitiva global. Esse caso ilustra padrões de alteração neuropsicológica em EH e a melhora destes com tratamento clínico.

Palavras-chave: Avaliação neuropsicológica; encefalopatia de Hashimoto; tireoidite de Hashimoto; funções cognitivas;
\end{abstract} neuropsicologia.

\section{Resumen}

La encefalopatía de Hashimoto (EH) es una condición neurológica rara, asociada a la presencia de altas concentraciones de anticuerpos anti-tiroideos y a la tiroiditis de Hashimoto. Este artículo presenta el caso de una paciente de 60 años con diagnóstico de EH, enfatizando su desempeño en tests neuropsicológicos antes y después del tratamiento medicamentoso. El estado de la paciente era saludable pero seis meses antes del diagnóstico comenzó a presentar depresión, crisis epilépticas, confusión mental, apraxias, mioclonías y alucinaciones visuales. Antes del tratamiento se le realizó una evaluación neuropsicológica que evidenció déficits cognitivos globales. Seis meses después del tratamiento fue reevaluada, mostrando recuperación cognitiva global. El presente caso ilustra el patrón de alteración neuropsicológica en la EH y su mejoría con tratamiento clínico.

Palabras-clave: Evaluación neuropsicológica; encefalopatía de Hashimoto; tiroiditis de Hashimoto; funciones cognitivas; neuropsicología.

\section{Résumé}

L'encéphalopathie d'Hashimoto $(\mathrm{EH})$ est une condition neurologique rare qui est associée à la présence d'anticorps antithyroïdiens et de la thyroïdite d'Hashimoto. Dans cet article, nous décrivons un cas clinique d'une patiente âgée de 60 ans, diagnostiquée comme présentant un $\mathrm{EH}$, en nous concentrant sur ses performances aux tests neuropsychologiques avant et après le traitement de sa maladie. Ce patient était préalablement en santé, mais six mois avant le diagnostique, elle présentait une dépression, des lésions épileptiques, une confusion mentale, une apraxie, une myoclonie et des hallucinations visuelles. Avant le traitement, une évaluation neuropsychologique montrait clairement un déficit cognitif

Artigo recebido: 29/03/2011; Artigo revisado: 12/04/2011; Artigo aceito: 28/04/2011.

Tatiana Q. Irigaray, Programa de Pós-graduação em Psicologia, Pontifícia Universidade Católica do Rio Grande do Sul. Michele Beckert, Universidade Federal do Rio Grande do Sul. Angélica D. Pizzol, Universidade Federal do Rio Grande do Sul. Candice S. Holderbaum, Instituto de Psicologia, Universidade Federal do Rio Grande do Sul. Denise R. Fontoura, Instituto de Psicologia, Universidade Federal do Rio Grande do Sul. Thirzá B. Frison, Universidade Federal do Rio Grande do Sul. Carlo M. Mattiello, Universidade Federal do Rio Grande do Sul. Taís P. Fachinelli, Universidade Federal do Rio Grande do Sul. Márcia L. F. Chaves, Faculdade de Medicina, Universidade Federal do Rio Grande do Sul. Marino M. Bianchin, Faculdade de Medicina, Universidade Federal do Rio Grande do Sul. Jerusa F. Salles, Instituto de Psicologia, Universidade Federal do Rio Grande do Sul.

Correspondências relacionadas a esse artigo devem ser enviadas a: Tatiana Quarti Irigaray, Avenida João Pessoa, ${ }^{\circ}$. 809, sala 103, Cidade Baixa, Porto Alegre, RS, CEP 90040-000.

E-mail: tatiana.irigaray@ superig.com.br

DOI: $10.5579 / \mathrm{rnl} .2011 .0058$ 
global. Six mois après le traitement, un nouveau suivi neuropsychologique a montré une récupération cognitive globale. Ce cas illustre la variance des patrons neuropsychologiques dans l'HE et les améliorations de ces altérations grâce au traitement clinique.

Mots-clès : Évaluation neuropsychologique ; encéphalopathie d'Hashimoto ; thyroïdite d'Hashimoto ; fonctions cognitives ; neuropsychologie.

\section{Abstract}

The Hashimoto's encephalopathy (HE) is a rare neurological condition that is associated with the presence of high concentrations of anti-thyroid antibodies and with Hashimoto's thyroiditis. In this article, we reported a clinical case of a 60 years old patient, diagnosed with HE, emphasizing her performance on neuropsychological tests before and after the treatment. The patient was previously healthy, but six months before the diagnosis, started with depression, epileptic seizures, mental confusion, apraxia, myoclonus and visual hallucination. Before treatment, she was submitted to a neuropsychological evaluation which showed clearly global cognitive deficit. Six months after treatment, she was submitted to a follow-up evaluation which showed a global cognitive recovery. This case illustrates patterns of neuropsychological variance on $\mathrm{HE}$ and the improvements of these changes with the clinical treatment.

Keywords: Neuropsychological assessment; Hashimoto's encephalopathy; Hashimoto's thyroiditis; cognitive functions; neuropsychology.

A encefalopatia de Hashimoto (EH) é uma condição neurológica rara que foi descrita por Brain, Jellinek e Ball em 1966 e está associada à presença de altas concentrações de anticorpos anti-tireoideanos e à tireoidite de Hashimoto (Cerqueira, Bezerra, Magalhães, Rozenthal, \& Nardi, 2008). Desde então, cerca de 100 casos foram descritos na literatura (Aquino, \& Mutarelli, 2009). Segundo Archambeuad et al. (2001), as encefalopatias associadas à tireoidite de Hashimoto são raras, porém sua existência ainda é provavelmente subestimada. A EH acomete predominantemente mulheres na quarta década de vida, com média de idade de 44 anos, sendo que $90 \%$ dos casos descritos na literatura afetam o sexo feminino (Aquino, \& Mutarelli, 2009; Archambeaud et al., 2001; Luque, Pomar, \& Echebarria, 2002).

A etiopatogenia da EH é desconhecida e seu diagnóstico é feito baseado em altos níveis de suspeita clínica, exclusão de infecções e de outros transtornos de origem metabólica, tóxica ou paraneoplásica (Aquino \& Mutarelli, 2009), além da presença de anticorpos anti-tireoideanos no sangue ou no líquor (Luque et al., 2002). O quadro de sintomas neurológicos compreende alteração do nível da consciência, sinais focais, crises epilépticas focais ou generalizadas, estado confusional, mioclonias, ataxias, tremores, transtornos psiquiátricos, demência e alterações motoras (Creutzfeldt, \& Haberl, 2005).

Existem dois tipos mais característicos de apresentação clínica, que podem ser sobrepostos. O primeiro é o tipo vasculítico de EH, que é caracterizado por episódios vasculares pseudoictais, que podem resultar em déficits neurológicos focais de forma aguda ou subaguda (Luque et al., 2002). O segundo tipo, conhecido como forma difusa progressiva da $\mathrm{EH}$, é caracterizado por declínio cognitivo progressivo, podendo levar a demência ou alterações psiquiátricas, tais como depressão, alucinações, confusão mental, sonolência e coma (Cerqueira et al., 2008; Luque et al., 2002).

Archambeaud et al. (2001) descreveram quatro pacientes com tireoidite de Hashimoto que apresentavam manifestações neuropsiquiátricas. Tratava-se de três mulheres e um homem, com média de idade de 68 anos, com epilepsia, mioclonias, episódios psicóticos, alteração da consciência e alucinações. Os exames neurológicos auxiliares de rotina, tais como eletroencefalograma (EEG) e ressonância magnética foram inespecíficos. Em todos os casos, os pacientes apresentaram tireoidite de Hashimoto com anticorpo antitireoperoxidase fortemente positivo e hipotireoidismo moderado. Os autores relataram melhora desses sintomas com uso de corticóides.

Cummings et al. (2007) relataram o caso de um homem com 19 anos, sem história neuropsiquiátrica prévia, que começou a apresentar confusão mental, desorientação e agitação 30 dias antes da hospitalização. Antes da confirmação do diagnóstico de $\mathrm{EH}$, foi feita a primeira avaliação neuropsicológica que revelou um comprometimento cognitivo global com déficits incluindo o estado mental global, a atenção simples e complexa, o raciocínio não verbal, a orientação, a aprendizagem de listas de palavras, de histórias, de figuras e de recordação. Durante a segunda hospitalização, 20 dias após a primeira, o paciente mostrou melhora no estado mental, na orientação e na recordação das listas de palavras. Depois de quatro meses de acompanhamento, ele mostrou melhora no estado mental, na orientação, na recordação de listas e na aprendizagem de listas de palavras, na codificação, no reconhecimento de listas de palavras e na recordação de figuras.

Aquino e Mutarelli (2009) apresentaram o caso de uma mulher com 42 anos, de São Paulo (Brasil) que, seis meses antes da hospitalização, demonstrava alteração de comportamento e agitação, tendo realizado tratamento psiquiátrico. $\mathrm{Na}$ admissão hospitalar, a paciente apresentava sintomas neuropsiquiátricos associados à ataxia, tremores e a mioclonias. A paciente teve o diagnóstico de $\mathrm{EH}$ e realizou pulsoterapia com metilprednisolona (1 g ao dia por 3 dias), obtendo um resultado excelente. $\mathrm{O}$ tratamento foi mantido com prednisolona oral. Dois anos depois, ela estava bem, usando prednisona $2,5 \mathrm{mg}$ em dias alternados e levotiroxina.

Creutzfeldt e Haberl (2005) descreveram o caso de uma mulher de 79 anos que apresentava dificuldades de concentração há três semanas, desorientação no tempo e espaço e auto-percepção de seu quadro. O tratamento foi iniciado com altas doses de corticóides e, após três dias de uso, a paciente estava caminhando e apresentando melhor estado mental. Em paralelo com a evolução clínica favorável, houve melhora importante no EEG. Três meses após a admissão hospitalar, apesar de a paciente apresentar progressos significativos, ela ainda demonstrava déficits cognitivos especialmente na memória de curto prazo.

O presente estudo tem por objetivo descrever um caso de encefalopatia de Hashimoto, caracterizando o perfil neuropsicológico da paciente no início e após seis meses de tratamento. 


\section{Caso}

\section{História clínica}

Uma mulher de 60 anos, branca, casada, destra, com a $5^{\mathrm{a}}$ série do Ensino Fundamental, natural e procedente do Rio Grande do Sul, trabalhava como caixa e fazia compras de mercadorias no estabelecimento comercial de sua família. Seis meses antes de sua transferência para o hospital, no qual foi feita a primeira avaliação neuropsicológica, a família começou a perceber que a paciente estava apresentando mudanças de comportamento. Ela estava falando menos, mostrava-se apática e apresentava sonolência excessiva. Começou também a cometer erros frequentes no seu trabalho, e estes eram especialmente evidentes durante as transações comerciais. Esses sintomas se agravaram progressivamente e, um mês após, ela começou a apresentar crises convulsivas. Ela foi, então, internada em uma instituição psiquiátrica e iniciou tratamento com carbamazepina, antidepressivos e antipsicóticos. Após a alta, a paciente já não caminhava sozinha, apresentava ansiedade, desorientação, delírios, crises de agitação psicomotora, discurso incoerente e dificuldade em reconhecer os familiares. O quadro foi se acentuando e ela foi novamente internada na mesma clínica psiquiátrica, na qual permaneceu por mais 50 dias, tendo recebido o diagnóstico de Demência associada a Corpos de Lewy. Após esse período ela foi transferida para o Hospital de Clínicas de Porto Alegre (HCPA).

No seu ingresso ao hospital, a paciente apresentava um quadro caracterizado por depressão, ansiedade, epilepsia, confusão mental, apraxias, mioclonias e alucinações visuais. Foi submetida a uma tomografia de encéfalo e ressonância nuclear magnética (RNM), que apresentaram resultados normais. O EEG apresentava-se moderadamente desorganizado à custa de aumento da atividade lenta, predominante nas áreas posteriores. Foram evidenciadas também raras descargas epileptiformes multifocais. A paciente foi submetida a uma extensa avaliação para doenças infecciosas, imunológicas e distúrbios metabólicos. Os anticorpos antiperoxidase (TPO) estavam elevados $(598,9)$ e a antitireoglobulina reagente (1/1600). A dosagem dos hormônios da tireóide estava em níveis normais. Baseado no quadro clínico e nos resultados dos exames auxiliares, foi estabelecido o diagnóstico de Encefalopatia de Hashimoto. A paciente foi tratada por um neurologista, inicialmente com pulsoterapia com metilprednisolona, com rápida melhora. Após o tratamento, foi mantido prednisona em doses decrescentes até $20 \mathrm{mg} / \mathrm{dia}$. A paciente recebeu alta hospitalar, com rápida melhora do quadro clínico e seguiu em acompanhamento ambulatorial.

A realização deste estudo, assim como a divulgação dos resultados, foram autorizadas pela paciente e pela familiar responsável. Ambas assinaram um Termo de Consentimento Livre e Esclarecido.

\section{Avaliações Neuropsicológicas}

A paciente realizou duas avaliações neuropsicológicas, sendo a primeira durante a internação e antes do início do tratamento medicamentoso e, a segunda, seis meses após ter sido medicada de forma adequada. Conforme descrito a seguir, a primeira avaliação neuropsicológica foi realizada no leito por uma psicóloga e uma fonoaudióloga, durante duas sessões, com duração média de 60 minutos cada, com aplicação de cinco instrumentos. A segunda avaliação neuropsicológica foi realizada pelas mesmas profissionais, durante 5 sessões, com duração média de 60 minutos cada. Nessa avaliação, aplicaram-se três instrumentos, utilizados na primeira avaliação, e foram acrescentados outros oito novos instrumentos com o intuito de realizar uma avaliação neuropsicológica mais completa.

\section{Primeira Avaliação Neuropsicológica}

$\mathrm{Na}$ primeira avaliação neuropsicológica, foram aplicados os seguintes instrumentos: Instrumento de Avaliação Neuropsicológica Breve NEUPSILIN (Fonseca, Salles, \& Parente, 2009), Teste Wisconsin de Classificação de Cartas (WCST) (Cunha et al., 2005), Token Test (Fontanari, 1989), Teste de Boston (compreensão auditiva, execução de ordens e material ideativo complexo) (Goodglass, \& Kaplan, 1983) e o subteste dígitos (ordem direta e ordem inversa) da Escala de Inteligência Wechsler para Adultos (Wechsler, 1997). Nessa avaliação, o NEUPSILIN foi utilizado com o objetivo de se obter uma avaliação breve do estado cognitivo geral da paciente. Durante a aplicação desse teste, as profissionais suspeitaram sobre uma possível dificuldade de compreensão verbal por parte da paciente. Então, decidiu-se aplicar testes para avaliar tal função. Para isso usou-se, o Token Test e o Teste de Boston). Além disso, suspeitou-se de déficits executivos, atencionais e na memória de trabalho, por isso, foram aplicados o WCST e o subteste Dígitos (ordem direta e inversa). Em virtude da paciente se encontrar no leito, não foram utilizados outros testes neuropsicológicos nessa primeira avaliação.

O NEUPSILIN (Fonseca et al., 2009) é um instrumento de avaliação neuropsicológica breve, porque possui um tempo reduzido de aplicação (entre 30 e 40 minutos) e inclui 32 tarefas que avaliam oito funções cognitivas e possui um tempo reduzido de aplicação (entre 30 e 40 minutos). As tarefas são curtas, avaliando orientação têmporo-espacial, atenção, percepção, memória (memória de trabalho, memória verbal: evocação imediata, evocação tardia e reconhecimento, memória semântica de longo prazo, memória visual de curto prazo, memória prospectiva), habilidades aritméticas, linguagem (linguagem oral e linguagem escrita), praxias (ideomotora, construtiva e reflexiva) e funções executivas (resolução de problemas e fluência verbal).

Conforme se pode verificar na Tabela 1, nessa primeira avaliação, realizada antes do tratamento medicamentoso, com o NEUPSILIN, a paciente apresentou déficits, que correspondem ao escore $\mathrm{Z} \leq-1,5$ desvio padrão, nas seguintes funções cognitivas: 1) percepção: reconhecimento de faces; 2) memória de trabalho: ordenamento ascendente de dígitos; 3) memória semântica de longo prazo; 4) memória visual de curto prazo; 5) habilidades aritméticas; 6) linguagem oral: processamento de inferências; 7) linguagem escrita: escrita ditada; 8) praxia reflexiva e; 9) funções executivas: número de vocábulos evocados. As outras funções cognitivas, avaliadas através do NEUPSILIN, encontravam-se preservadas.

O WCST (Cunha et al., 2005) é uma medida das funções executivas e requer a capacidade do examinando para desenvolver e manter uma estratégia apropriada de solução de problema por meio de condições de estímulos mutáveis a fim de atingir uma meta futura. É constituído por quatro cartasestímulo e 128 cartas-resposta, que representam figuras de 
variadas formas, cores e número. Nessa primeira avaliação neuropsicológica, no WCST, a paciente completou duas categorias (percentil 6-10), usou 40 ensaios para completar a primeira categoria (percentil $\leq 1)$ e um escore de aprendendo

Tabela 1

Escore Z da paciente no NEUPSILIN na primeira e na segunda avaliação neuropsicológica e interpretação dos resultados

\begin{tabular}{|c|c|c|c|}
\hline NEUPSILIN & $\begin{array}{l}1^{\text {a }} \text { Avaliação } \\
\text { Escore Z }\end{array}$ & $\begin{array}{l}2^{\mathrm{a}} \text { avaliação } \\
\text { Escore } \mathrm{Z}\end{array}$ & Interpretação \\
\hline Orientação temporal & $+0,42$ & $+0,42$ & Nenhuma \\
\hline Orientação espacial & $+0,14$ & $+0,14$ & Nenhuma \\
\hline Atenção: Contagem Inversa & $-0,50$ & $-1,79 *$ & $1>2$ \\
\hline Atenção: Repetição de dígitos & $+0,16$ & $+0,16$ & Nenhuma \\
\hline $\begin{array}{l}\text { Percepção: Verificação de igualdades e } \\
\text { diferenças de linhas }\end{array}$ & $+0,84$ & $+0,84$ & Nenhuma \\
\hline Percepção: heminegligência visual & 0 & 0 & Nenhuma \\
\hline Percepção: percepção de Faces & $-0,33$ & $-0,33$ & Nenhuma \\
\hline Percepção: reconhecimento de Faces & $-1,64 *$ & $+0,48$ & $1<2$ \\
\hline $\begin{array}{l}\text { Memória de Trabalho: Ordenamento } \\
\text { Ascendente de Dígitos }\end{array}$ & $-2,44^{*}$ & $-0,17$ & $1<2$ \\
\hline $\begin{array}{l}\text { Memória de Trabalho: Span Auditivo de } \\
\text { Palavras em Sentenças }\end{array}$ & $-0,22$ & $-0,90$ & $1>2$ \\
\hline Memória Verbal: Evocação Imediata & $-0,29$ & $+1,23$ & $1<2$ \\
\hline Memória Verbal: Evocação Tardia & $+0,46$ & $-0,77$ & $1>2$ \\
\hline Memória Verbal: Reconhecimento & $-0,45$ & $+1,87$ & $1<2$ \\
\hline Memória Semântica de Longo Prazo & $-3,77 *$ & $+0,48$ & $1<2$ \\
\hline Memória Visual de Curto Prazo & $-2,67 *$ & $-1,0$ & $1<2$ \\
\hline Memória Prospectiva & $-0,33$ & $-0,33$ & Nenhuma \\
\hline Habilidades aritméticas & $-1,52 *$ & $+0,69$ & $1<2$ \\
\hline Linguagem oral: nomeação & 0 & 0 & Nenhuma \\
\hline Linguagem oral: repetição & $+0,45$ & $+0,45$ & Nenhuma \\
\hline Linguagem oral: automática & $+0,21$ & $+0,21$ & Nenhuma \\
\hline Linguagem oral: compreensão & $+0,45$ & $+0,45$ & Nenhuma \\
\hline Linguagem oral: inferências & $-1,89^{*}$ & $-1,89 *$ & Nenhuma \\
\hline Linguagem escrita: leitura voz alta & $+0,55$ & $+0,59$ & $1<2$ \\
\hline Linguagem escrita: compreensão escrita & $-1,36$ & $+0,45$ & $1<2$ \\
\hline Linguagem escrita: escrita espontânea & 0,60 & 0,60 & Nenhuma \\
\hline Linguagem escrita: escrita copiada & 0,70 & 0,70 & Nenhuma \\
\hline Linguagem escrita: escrita ditada & $-2,39 *$ & $-1,23$ & $1<2$ \\
\hline Praxia idemomotora & $+0,14$ & $+0,14$ & Nenhuma \\
\hline Praxia construcional & $+0,58$ & $+0,15$ & $1<2$ \\
\hline Praxia reflexiva & $-1,64 *$ & $-0,72$ & $1<2$ \\
\hline Funções executivas: resolução de problemas & $-1,03$ & $-1,03$ & Nenhuma \\
\hline $\begin{array}{l}\text { Funções executivas: número de vocábulos } \\
\text { evocados }\end{array}$ & $-1,51^{*}$ & $-0,24$ & $1<2$ \\
\hline
\end{tabular}

Nota. * déficits

a aprender de -13,75 (percentil 6-10), demonstrando escores inferiores ao esperado para sua idade e escolaridade.

O Token Test (Fontanari, 1989) avalia a compreensão oral para comandos de complexidade crescente. O examinando deve executar ordens simples. $\mathrm{O}$ escore obtido pela paciente, na primeira avaliação, no Token Test, foi de 28 pontos, que corresponde a um comprometimento leve na compreensão da linguagem.

O Teste de Boston (Goodglass, \& Kaplan, 1983) avalia a linguagem, sendo composto por várias tarefas que medem a compreensão e a emissão oral e gráfica. Nessa avaliação, utilizou-se as tarefas de compreensão auditiva, execução de ordens e material ideativo complexo. Nas duas primeiras, a paciente apresentou escores normais. No entanto, na avaliação de material ideativo complexo, obteve desempenho abaixo do esperado para sua idade e escolaridade.
O subteste dígitos (ordem direta e ordem inversa) da Escala de Inteligência Wechsler para Adultos (Wechsler, 1997) avalia principalmente a capacidade de memória de trabalho (auditiva) e atenção. As duas tarefas desse subteste envolvem a repetição de números apresentados oralmente pelo examinador, sendo a tarefa solicitada na ordem inversa nitidamente com maior grau de complexidade. Nas duas tarefas avaliadas, a paciente obteve desempenho abaixo do esperado para sua idade e escolaridade.

\section{Segunda Avaliação Neuropsicológica}

A segunda avaliação neuropsicológica foi realizada seis meses após tratamento medicamentoso e foi aplicado um total de onze instrumentos. Destes, três foram os mesmos aplicados na primeira avaliação (NEUPSILIN, WCST e dígitos). Os outros instrumentos utilizados foram os seguintes: Mini-exame do Estado Mental (MEEM) (Brucki, Nitrini, 
Caramelli, Bertolucci, \& Okamoto, 2003); Escala de Inteligência Wechsler para adultos (Wechsler, 1997): subtestes completar figuras, vocabulário, cubos, raciocínio matricial, aritmética e semelhanças; Teste de aprendizagem auditivo-verbal de Rey (RAVLT) (Diniz et al., 2000); Teste de Reprodução Visual de Figuras da Escala de Memória de Wechsler Revisada (Strauss, Sherman, \& Spreen, 2006); Teste de Memória Lógica da Escala de Memória de Wechsler Revisada (Strauss, Sherman, \& Spreen, 2006); Teste de Atenção Concentrada - AC (Cambraia, 2004); Escala de atividades de vida diária (Katz, Downs, Cash, \& Grotz, 1970) e; Escala de atividades instrumentais de vida diária (Lawton, Moss, Fulcomer, \& Kleban, 1982).

A Tabela 1 mostra os escores $Z$ obtidos no NEUPSILIN (Fonseca et al., 2009) nesta segunda avaliação neuropsicológica e também a interpretação desses resultados. Conforme se pode observar, a paciente apresentou melhora nas seguintes funções cognitivas: 1) Percepção: reconhecimento de Faces; 2) Memória de Trabalho: Ordenamento Ascendente de Dígitos; 3) Memória Verbal: Evocação Imediata; 4) Memória Verbal: Reconhecimento; 5) Memória Semântica de Longo Prazo; 6) Memória Visual de Curto Prazo; 7) Habilidades aritméticas; 8) Linguagem escrita: leitura voz alta; 9) Linguagem escrita: compreensão escrita; 10) Linguagem escrita: escrita ditada; 11) Praxia construcional 12) Praxia reflexiva; e 13) Funções executivas: número de vocábulos evocados. Mostrou desempenho que pode ser considerado indicativo de déficits apenas nas funções de: 1) atenção: contagem inversa; e 2) linguagem oral: processamento de inferências. Apresentou pior desempenho nas funções de 1) memória verbal: evocação tardia; 2) atenção: contagem inversa; e 3) memória de trabalho: span auditivo de palavras em sentenças. Nas demais funções cognitivas, avaliadas pelo NEUPSILIN, obteve escores iguais aos da primeira avaliação neuropsicológica. Nessa segunda avaliação, demonstrou melhor desempenho no WCST (Cunha et al., 2005) e no subteste dígitos (Wechsler, 1997) em relação à primeira. No WCST, completou três categorias (percentil $>16$ ), usou 18 ensaios para completar a primeira categoria (percentil 11-16) e um escore de aprendendo a aprender de 7,6 (percentil >16). No subteste dígitos, obteve $11 \%$ de acertos a mais em relação à primeira avaliação.

O MEEM (Brucki, Nitrini, Caramelli, Bertolucci, \& Okamoto, 2003) é um instrumento de avaliação global das funções cognitivas. É composto por questões que avaliam orientação para tempo, orientação para local, registro de três palavras, atenção e cálculo, lembrança de três palavras, linguagem e capacidade construtiva visual. A pontuação pode variar de zero até 30 pontos. $\mathrm{O}$ escore obtido pela paciente no MEEM foi de 24 pontos, que representa uma pontuação normal para sua escolaridade. Perdeu 3 pontos no sete seriado, 2 pontos na evocação do nome dos objetos e um ponto na tarefa de seguir uma instrução.

Os subtestes completar figuras, raciocínio matricial, aritmética, semelhanças, cubos e vocabulário da Escala de Inteligência Wechsler para adultos (Wechsler, 1997) foram aplicados para caracterização do desempenho em tarefas de memória visual, raciocínio não-verbal, cálculos, memória auditiva, raciocínio lógico, inteligência geral, destreza visuomotora, habilidades visuo-perceptivas e visuo-construtivas, entre outros processos cognitivos. Em todos esses subtestes, a paciente obteve escores abaixo do esperado para sua idade e escolaridade. Através dos subtestes vocabulário e cubos da
Escala de Inteligência Wechsler para adultos (Wechsler, 1997) foi calculado o Quociente Intelectual Estimado da paciente. Obteve-se uma pontuação total de 82 , que corresponde a um QI de nível médio inferior,

O RAVLT (Malloy-Diniz et al., 2000) mede a memória episódica verbal (memória recente), aprendizagem, interferência, retenção e memória de reconhecimento. O teste consiste de duas listas compostas por 15 substantivos cada, lidos num total de seis vezes, que o indivíduo deve recordar após a apresentação de cada lista. Nessa tarefa, a paciente não apresentou uma curva ascendente de aprendizagem, demonstrando dificuldades relacionadas à aprendizagem e retenção de conteúdo verbal (imediata e tardia). Em todas as tentativas de repetição das listas seu desempenho sempre foi abaixo do esperado para sua idade e escolaridade.

Teste de Reprodução Visual de Figuras da Escala de Memória de Wechsler Revisada (Strauss, Sherman, \& Spreen, 2006). É uma medida que caracteriza a memória visual (imediata e tardia), o planejamento e a função visoconstrutiva. $\mathrm{O}$ teste contém quatro figuras que são apresentadas individualmente para o participante, que deve reproduzir imediatamente após a apresentação de cada figura e depois de um intervalo de 30 minutos. Na evocação imediata das figuras, a paciente obteve escores dentro do padrão da normalidade, apresentando preservadas as funções de planejamento, organização, habilidade visuoespacial e memória visual de curto. Na evocação tardia, demonstrou desempenho abaixo do esperado para sua idade e escolaridade.

Teste de Memória Lógica da Escala de Memória de Wechsler Revisada (Strauss, Sherman, \& Spreen, 2006). É composta por duas histórias relatadas ao paciente e evocadas imediatamente após e depois de 30 minutos. Avalia a memória episódica verbal (imediata e tardia). Tanto na evocação imediata quanto tardia das duas histórias, a paciente obteve escores abaixo do esperado para sua idade e escolariadade.

As escalas de atividades de vida diária (Katz, Downs, Cash, \& Grotz, 1970) e atividades instrumentais de vida diária (Lawton, Moss, Fulcomer, \& Kleban, 1982) foram aplicadas com o objetivo de avaliar a capacidade funcional da paciente. Nas duas escalas, obteve pontuação considerada normal, demonstrando independência tanto para as atividades de vida diária quanto para as atividades instrumentais de vida diária.

\section{Discussão}

No presente caso, o diagnóstico de $\mathrm{EH}$ foi confirmado por que a paciente apresentava um quadro clínico caracterizado por depressão, ansiedade, epilepsia, confusão mental, apraxias, mioclonias e alucinações visuais, sem uma causa infecciosa, tumoral, vascular ou por doenças sistêmicas. Além disso, apresentava anticorpos antiperoxidase (TPO $598,9)$ e antitireoglobulinas (1/1600) positivos e demonstrou uma boa resposta ao uso de corticóide. Segundo Archambeaud et al. (2001), a dosagem plasmática de anticorpos anti-TPO na EH é sempre elevada, servindo como um marcador para a doença. Da mesma maneira, Aquino e Mutarelli (2009) afirmam que anticorpos anti-TPO e antitireoglobulina estão presentes em $95-100 \%$ e $73 \%$ dos casos de EH, respectivamente.

Segundo estudos, a maioria dos pacientes mostra uma excelente resposta ao tratamento com corticóides, que é 
também associada a uma redução importante da taxa de anticorpos anti-TPO (Archambeaud et al. 2001; Creutzfeldt, \& Haberl, 2005). Em casos de coma, a eficácia da corticoterapia é vista entre um a três dias e, em outras manifestações neurológicas, a resposta inicial pode ser rápida, com melhora progressiva ao longo das semanas subsequentes. A duração da corticoterapia varia de acordo com o quadro clínico do paciente (Archambeaud et al., 2001).

No caso relatado, a paciente apresentou melhora tanto neurológica quanto neuropsicológica com uso de corticóide. Em relação à primeira avaliação neuropsicológica, ela demonstrou, na segunda avaliação, uma melhora cognitiva global no Instrumento de Avaliação Neuropsicológica Breve NEUPSILIN, apresentando melhor desempenho em 13 tarefas e desempenho igual em 16. Na última avaliação, apresentou déficits apenas nos subtestes do NEUPSILIN de atenção (contagem inversa) e linguagem oral (processamento de inferências) e piora nas tarefas de memória verbal (evocação tardia), atenção (contagem inversa) e memória de trabalho (span auditivo de palavras em sentenças). O seu desempenho nas funções executivas (WCST) também melhorou em comparação à primeira avaliação. No entanto, ainda apresentava escores abaixo do esperado para sua idade e escolaridade em tarefas de atenção, memória, aprendizagem, recordação, raciocínio lógico-sequencial, destreza visuomotora, habilidades visuo-perceptivas e visuo-construtivas. Em tarefas de memória visual (evocação imediata) demonstrou desempenho normal. Em decorrência da melhora proporcionada pelo tratamento, a paciente retomou as suas atividades de trabalho e voltou a ser independente para as atividades de vida diária e atividades instrumentais de vida diária. Esses resultados são coerentes com dados da literatura que apontam que muitos dos pacientes retornam ao seu estado neurológico normal (Aquino \& Mutarelli, 2009, Archambeaud et al., 2001; Luque et al., 2002). No entanto, alguns sintomas podem persistir, tais como tremores e perda de memória (Aquino \& Mutarelli, 2009).

Cummings et al. (2007) sugerem o uso da avaliação neuropsicológica para auxiliar no diagnóstico de EH e no manejo da medicação, uma vez que existem dificuldades diagnósticas e dúvidas em relação ao tempo de tratamento com corticóide e a forma como deve ser administrado. Da mesma maneira, Creutzfeldt e Haberl (2005) afirmam que a avaliação neuropsicológica e o EEG são os instrumentos mais adequados para auxiliar no diagnóstico e monitoração do tratamento. Em um caso de EH relatado por Cummings et al. (2007), embora o paciente apresentasse melhora clínica, a avaliação neuropsicológica apontou déficits cognitivos que indicaram a necessidade de continuidade de tratamento com corticóide. Segundo os mesmos autores, é possível que a avaliação neuropsicológica, nesses casos, auxilie na identificação de um padrão de déficits suficientemente específicos que possam ser adicionados aos critérios diagnósticos.

O EEG frequentemente demonstra alterações, caracterizadas por atividade lenta e difusa, e tende a normalizar-se após a corticoterapia. A RNM, na maioria dos casos é normal, mas pode apresentar anormalidade do tipo sinal de aumento difuso na substância branca. Após a corticoterapia, as anormalidades apresentadas no EEG e na RNM tendem a desaparecer (Aquino, \& Mutarelli, 2009; Archambeaud et al., 2001; Luque et al., 2002).
Para Luque et al. (2002), a EH é uma condição ainda pouco diagnosticada. Isso talvez ocorra porque a doença ainda é pouco conhecida e compreendida, além de altos níveis de suspeita clínica serem necessários para um diagnóstico rápido e acurado. Desta forma, muitos autores sugerem que em todo o paciente de meia idade, em especial mulheres, com quadro de encefalopatia de causa desconhecida, deve-se suspeitar do diagnóstico de EH e verificar a dosagem dos anticorpos antitireoideanos de forma prematura (Luque et al., 2002).

O caso apresentando é similar a outros apresentados na literatura, com um quadro clínico sugestivo de comprometimento encefálico progressivo e global, com presença positiva de anticorpos antiperoxidase e antitireoglobulina. Verificou-se, também, que a paciente demonstrou uma boa resposta ao tratamento com corticóide, apresentando tanto uma melhora clínica quanto cognitiva, o que confirma o diagnóstico de EH. Além disso, ressalta-se a importância da avaliação neuropsicológica nesses casos como um método complementar para o diagnóstico e o planejamento de manejo do tratamento, uma vez que aponta as potencialidades e os déficits cognitivos do paciente, contribuindo para a decisão de continuidade ou não do tratamento.

\section{Referências}

Archambeaud, F., Galinat, S., Regouby, Y., Magy, L., Rebeyrotte, J. M., Vallat, J. M., \& Teissier, M. P. (2001). Encéphalopathie de Hashimoto. Analyse de quatre observations. La Revue de Médecine Interne, 22 (7), 653-659.

Aquino, R. T. R, \& Mutarelli, E. (2009). Haschimoto's encephalopathy. Arquivos de Neuro-psiquiatria, 67(3-A), 724-725.

Brain L., Jellinek, E. H., \& Ball, K. (1966). Hashimoto's disease and encephalopathy. Lancet, 2, 512-514.

Brucki, S. M., Nitrini, R., Caramelli, P., Bertolucci, P. H., \& Okamoto, I. H. (2003).Sugestões para o Uso do Mini-Exame do Estado Mental no Brasil. Arquivos de Neuropsiquiatria, 61(3-B), 777-781.

Cambraia, S. V. (2004). Teste de Atenção Concentrada. São Paulo: Vetor Editora Psicopedagógica Ltda.

Cerqueira, A. C. R., Bezerra, J. M. F., Magalhães, G. C., Rozenthal, M., \& Nardi, A. E. (2008). Hashimoto's encefalopatthy with clinical features similar to those of Creutzfeldt-Jakob disease. Arquivos de Neuropsiquiatria, 66(4), 903-905.

Creutzfeldt, C. J., \& Haberl, R. L. (2005). Hashimoto Encephalopathy: a do not-miss in the diagnosis of dementia. Journal of Neurology, 252(10), 1285-1287.

Cunha, J. A., Trentini, C. M., Argimon, I. L., Oliveira, M. S., Werlang, B.G., \& Prieb, R. G. (2005). Teste Wisconsin de Classificação de Cartas: manual. São Paulo: Casa do Psicólogo.

Cummings, R. R., Bagley, S. C., Syed, S., Dong, N., \& Lin, A. (2007). Hashimoto's encephalopathy: a case report with neuropsychological testing. General Hospital psychiatry, 29(3), 267- 269.

Diniz, L. F. M., Cruz, M. de F. da, Torres, V. de M., \& Cosenza, R. M. (2000). O teste de aprendizagem auditivo-verbal de Rey: normas para uma população brasileira. Revista brasileira de neurologia, 36(3), 7983.

Fonseca, R. P., Salles, J. F., \& Parente, M. A. M. P. (2009). Instrumento de avaliação neuropsicológica breve NEUPSILIN. São Paulo, SP: Vetor.

Fontanari, J. L. (1989). O "Token Test”: elegância e concisão na avaliação da compreensão do afásico. Validação da versão reduzida de De Renzi para o português. Neurobiologia. 1989; 52(3):177-218.

Goodglass H., \& Kaplan E. (1983). The Assessment of Aphasia and Related Disorders. 2nd edn. Lea \& Febiger, Philadelphia, PA, USA.

Katz, S., Down, T. D., Cash, H. R., \& Grotz, R.C. (1970). Progress in the development of the index of ADL. The Gerontologist, 10(1), 20-30.

Lawton, M. P., Moss, M., Fulcomer, M., \& Kleban, M. H. (1982). A research and service oriented multilevel assessment instrument. Journal of Gerontology, 37(1), 91-99.

Luque, A. A., Pomar, M. D. B., \& Echebarría, L. H. (2002). Encefalopatía de Hashimoto. Neurologia, 17(10): 628-632.

Strauss, E., Sherman, E. M. S., Spreen, O. (2006). A Compendium of Neuropsychological Tests: Administration, Norms and Commentary. New York: Oxford University Press. 
PERFIL NEUROPSICOLÓGICO ENCEFALOPATIA DE HASHIMOTO

Sue, C. M, Fung, V., Halpern, J. P., Boyages, S. C., \& Yiannikas, C. (1997). Hashimoto's encephalopathy. Journal of Clinical Neuroscience, 4(1), 74-77.

Wechsler, D. (1997). WAIS-III: Escala de inteligência Wechsler para adultos - manual David Wechsler. Adaptação e padronização de uma amostra brasileira. Elizabeth do Nascimento, $\left(1^{a}\right.$ ed.). São Paulo: Casa do Psicólogo. 